\title{
Paper
}

\section{Novel Iron Oxides with Square Planar Coordination from Low Temperature Synthesis}

\author{
Cédric Tassel, Yoshihiro Tsujimoto, Hiroshi Kageyama and Kazuyoshi Yoshimura \\ Department of Chemistry, Graduate School of Science, Kyoto University, Kitashirakawa Oiwake-cho Sakyo-ku, Kyoto 606-8502, Japan.
}

Received November 4, 2009

\section{SYNOPSIS}

By using new synthetic approaches, one can create new families of materials which could lead to new chemical and physical properties. From this philosophy, we have synthesized novel iron oxides by topochemically extracting oxygen from well known perovskites derivatives $\mathrm{SrFeO}_{3}$, layered $\mathrm{Sr}_{3} \mathrm{Fe}_{2} \mathrm{O}_{7}$ and brownmillerite $\mathrm{CaFeO}_{2.5}$. The new materials $\mathrm{SrFeO}_{2}, \mathrm{Sr}_{3} \mathrm{Fe}_{2} \mathrm{O}_{5}$ and $\mathrm{CaFeO}_{2}$ possess $\mathrm{FeO}_{4}$ square planes and derivatives. These novel materials show new structures in which exotic electronic and magnetic properties can be witnessed. They also provide clues to understand oxygen diffusion in solids. In this review, we describe the chemical and physical properties of these novel iron oxides.

\section{KEY WORDS}

Perovskites, infinite layer, square planar coordination, iron oxides, hydride reduction, soft-chemistry

\section{Introduction}

Low temperature reactions such as intercalation and desintercalation allow the rational design of structures by preserving the main framework of a given compound, leading to the modification of coordination. This is in marked contrast with high temperature synthesis which limits the control of coordinations to those within known geometries. Iron is one of the most abundant metals on earth and forms numerous oxides, some of which are used in industry for pigments and ferrite magnets. In almost all of these oxides, iron bonds tetrahedrally, pyramidally or octahedrally with oxygen atoms. By using a low temperature synthesis approach we have been able to synthesize new materials containing iron in an oxygen square planar coordination.

\section{$2 \mathrm{SrFeO}_{2}$}

$\mathrm{SrFeO}_{3-\delta}(0 \leq \delta \leq 0.5)$ (Fig. 1a) has been intensively studied because it exhibits, for example, fast oxygen transport combined with high electron conductivity at low temperatures. It is thus a potential candidate for electrodes for solid oxide fuel cells and batteries ${ }^{1)}$, membranes for oxygen separation ${ }^{2)}$, catalysis ${ }^{3)}$ and gas sensors ${ }^{4}$. The $\delta=0.5$ phase (Fig. 1b) was the reduction limit of this compound despite extensive attempts to control the oxygen content including synthesis at high temperature with oxygen partial pressure ranging from $10^{-9}$ to $100 \mathrm{MPa}^{5,6)}$, electrochemical reactions ${ }^{7}$, fabrication epitaxial thin films under different atmospheres ${ }^{8)}$.

Our approach follows recent success in the use of hydrides of electropositive metals as powerful reducing agents (e.g. $\mathrm{CaH}_{2}$ and $\mathrm{NaH}$ ). For instance, reaction with $\mathrm{NaH}$ of $\mathrm{LaSrCoO}_{4}$ and $\mathrm{Yb}_{2} \mathrm{Ti}_{2} \mathrm{O}_{7}$ yielded $\mathrm{LaSrCoO}_{3.38}{ }^{9)}$ and $\mathrm{Yb}_{2} \mathrm{Ti}_{2} \mathrm{O}_{6.43}{ }^{10)}$, respectively. Here, we have been able to overcome the $\delta=0.5$ limit of $\mathrm{SrFeO}_{3-\delta}$ to form $\mathrm{SrFeO}_{2}{ }^{11)}$. This structure was synthesized by mixing $\mathrm{SrFeO}_{3}$ with $\mathrm{CaH}_{2}(1: 2$ molar ratio) and heating the mixture under vacuum at $280^{\circ} \mathrm{C}$ for one day. The pseudo-cubic phase $(a \approx 3.86 \AA)$ transforms to a tetragonal structure $(a=3.9911$ (a)

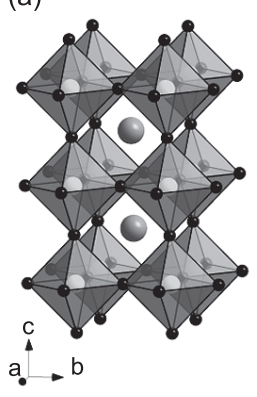

(b)

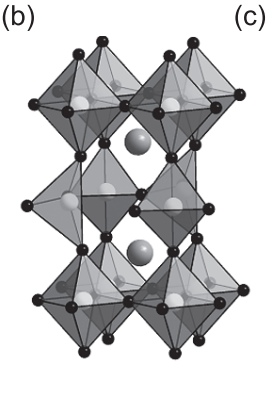

(c)

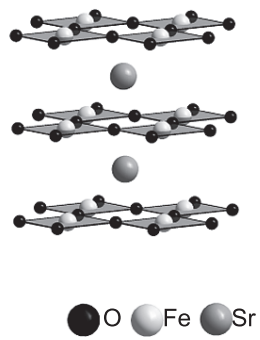

Fig.1 Schematic structure of the perovskite $\mathrm{SrFeO}_{3}$ (a), the brownmillerite $\mathrm{SrFeO}_{2.5}$ (b) and the infinite layer $\mathrm{SrFeO}_{2}$ (c). 
$\AA$ and $c=3.4748 \AA$ ). The $\mathrm{x}$-ray pattern (Fig. 2) revealed a $P 4 / \mathrm{mmm}$ space group, which is the same as the infinite layer compound $\mathrm{SrCuO}_{2}$. The stoichiometry $(1: 1)$ between strontium and iron was checked by EDX, and thermogravimetric measurement showed an oxygen stoichiometry of 2 in agreement with $\delta=1$. Rietveld analysis from both $\mathrm{x}$-ray and neutron diffraction confirmed that the structure is isostructural with $\mathrm{SrCuO}_{2}$ (Fig. 1c). Thus, the structure can be described has an infinite layered structure with iron bearing a square planar oxygen coordination. There was only one report on such a coordination, the gillepsite $\mathrm{BaFeSi}_{4} \mathrm{O}_{10}{ }^{12)}$. $\mathrm{CaFe}_{3} \mathrm{Ti}_{4} \mathrm{O}_{12}$ and $\mathrm{CaFeTi}_{2} \mathrm{O}_{6}$ were claimed to possess $\mathrm{FeO}_{4}$ square planes but there are four long $\mathrm{Fe}-\mathrm{O}$ bonds and two short $\mathrm{Fe}-\mathrm{O}$ bonds ${ }^{13)}$.

Iron in $\mathrm{SrFeO}_{2}$ is bivalent and in the high spin state $(S=2)$. This compound is magnetically ordered at room temperature having a $G$-type antiferromagnetic structure with the spin lying onto the $a b$ plane. Despite its low dimensional structure, the Néel temperature appears to be fairly high $(473 \mathrm{~K})$. This is due to the strong interactions in the $a b$ plane as well as in between vertical Fe $\cdots \mathrm{Fe}$. The $\left(d_{x z}\right.$, $\left.d_{y z}\right)^{3}\left(d_{x y}\right)^{1}\left(d_{z^{2}}\right)^{1}\left(d_{x^{2}-y^{2}}\right)^{1}$ electronic configuration was first suggested, in reason of small quadrupole splitting values possibly indicating a degenerated ground state. However, Jahn-Teller distortion has not been observed at low temperature. Further investigations using density functional theory, band structure and total energy calculations revealed that the $d_{z 2}$ level has the lowest energy level and is doubly occupied due to lower Coulomb repulsion and a possible intra-atomic mechanism associated with the hybridization of $d_{z^{2}}$ with the $4 s$ energy level. The resulting electronic configuration $\left(d_{z^{2}}\right)^{2}\left(d_{x z}, d_{y z}\right)^{2}\left(d_{x y}\right)^{1}\left(d_{x 2-y 2}\right)^{1}$ explains the absence of structural transitions at low temperatures

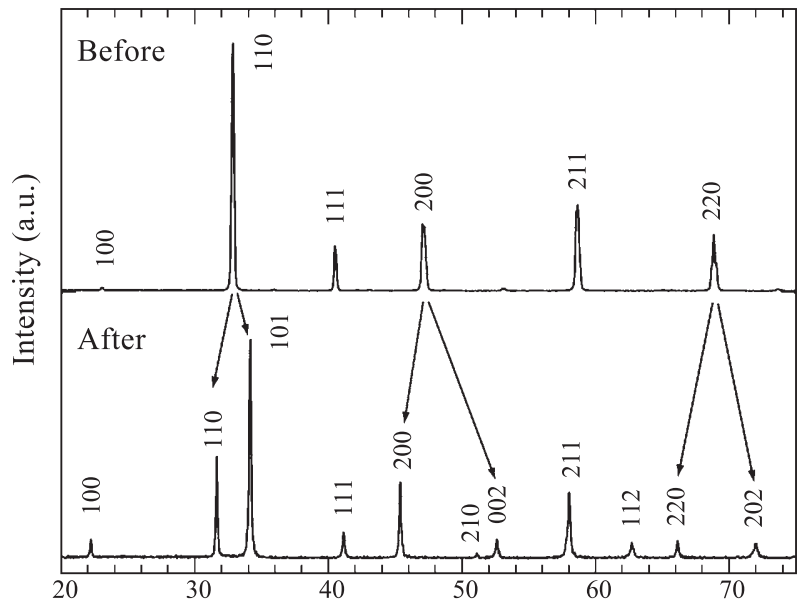

Fig.2 XRD patterns of $\mathrm{SrFeO}_{3}$ (up) and $\mathrm{SrFeO}_{2}$ (down). distortions as well as the observed magnetic structure ${ }^{14,15)}$.

The high pressure investigation of $\mathrm{SrFeO}_{2}$ led to the discovery of a spin transition to the intermediate state $S=1$ accompanied by a transition from an antiferromagnetic insulating state to a ferromagnetic half metal ${ }^{16)}$. The intermediate spin state is rare for $d^{6}$ elements and occur mostly in five-fold coordinated compounds (e.g. $\mathrm{TbBaCo}_{2} \mathrm{O}_{5}$ with a pyramidal coordination), moreover spin transition has never been observed in tetra-coordinated metal. At the critical pressure of $33 \mathrm{GPa}, \mathrm{SrFeO}_{2}$ decreases its volume by $3 \%$ and the electronic configuration of iron changes from $\left(d_{z^{2}}\right)^{2}\left(d_{x z}, d_{y z}\right)^{1}\left(d_{x y}\right)^{1}\left(d_{x^{2}-y^{2}}\right)^{1}\left(=d^{6}\right)$ to an exotic $\left(d_{z^{2}}\right)^{2}\left(d_{x z}, d_{y z}\right)^{3}\left(d_{x y}\right)^{1}\left(d_{x 2-y 2}\right)^{0.5}\left(=d^{6.5}\right)$ state due to the strong in-layer hybridization between $\mathrm{Fe}-d_{x^{2}-y^{2}}$ and $\mathrm{O}-p_{\sigma}$ bonding states, leading to an electronic instability towards depopulation of $\mathrm{Fe}-d_{x^{2}-y^{2}}$ and $\mathrm{O}-p_{\sigma}$ bonding states. The transition to a half-metallic state is of high interests and provides a hope that higher pressure could lead to superconductivity. Much effort to reduce the pressure transition has to be done and the study of other structures containing square planes could lead to this result.

Hydride reduction was later on applied for the first time to thin film of $\mathrm{SrFeO}_{3}$ by Inoue and coworkers ${ }^{17)}$ who synthesized epitaxial films of $\mathrm{SrFeO}_{2}$. Such reaction applied to thin film opens new doors towards possible applications. Recently, Ju et al. predicted a strong optical anisotropy in $\mathrm{SrFeO}_{2}$ and suggested applications in optoelectronics ${ }^{18)}$.

\section{$3 \mathrm{Sr}_{3} \mathrm{Fe}_{2} \mathrm{O}_{5}$}

Following the synthesis of the infinite layer structure, $\mathrm{Sr}_{3} \mathrm{Fe}_{2} \mathrm{O}_{5}$ was synthesized from the slightly oxygen deficient layered perovskite compound $\mathrm{Sr}_{3} \mathrm{Fe}_{2} \mathrm{O}_{7-\delta}$ (Fig. 3) ${ }^{19}$. The structure of $\mathrm{Sr}_{3} \mathrm{Fe}_{2} \mathrm{O}_{7}$ consists of double sheets of $\mathrm{SrFeO}_{3}$ perovskite separated by $\mathrm{SrO}$ rocksalt layers. In this phase, it is known that oxygen vacancies are located only at the apical $\mathrm{O}(1)$ site and the structure is kept tetragonal $(I 4 / \mathrm{mmm})$ for $0 \leq \delta \leq 1$. Using a similar method to $\mathrm{SrFeO}_{2}$, we were able to yield $\mathrm{Sr}_{3} \mathrm{Fe}_{2} \mathrm{O}_{5}$ (Fig. 3c). The new lattice is orthorhombic Immm ( $a=3.51485$ (2) $\AA, b=3.95271(2) \AA$, and $c=20.91251$ (10) $\AA$ ) (Fig. 4). Rietveld analysis of SXRD and neutron scattering showed that the original double perovskite layers become decoupled into ladders running along the $b$ axis. It was revealed that the reaction proceeds from $\mathrm{Sr}_{3} \mathrm{Fe}_{2} \mathrm{O}_{7}$ to $\mathrm{Sr}_{3} \mathrm{Fe}_{2} \mathrm{O}_{6}$ by the removal of the $\mathrm{O}$ (1) sites resulting in pyramidal planes and further reduction of the $\delta=1$ phase gives the removal of one oxygen of the $\mathrm{O}(2)$ site and the transfer of one oxygen to the $\mathrm{O}$ (1) site leading to $\mathrm{Sr}_{3} \mathrm{Fe}_{2} \mathrm{O}_{5}$. 
(a)
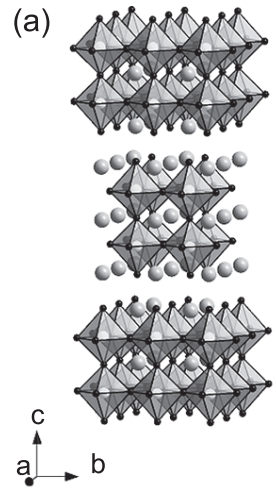

(b)

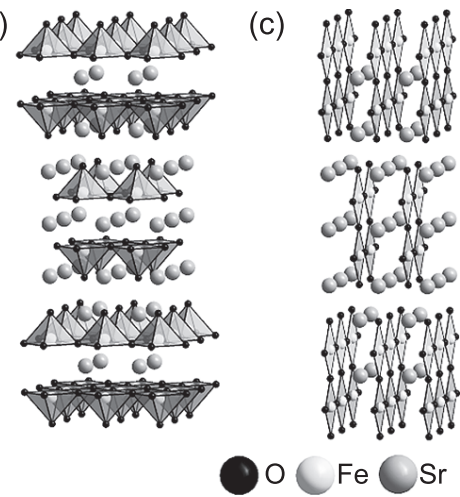

Fig.3 Structure of the layered perovskite $\mathrm{Sr}_{3} \mathrm{Fe}_{2} \mathrm{O}_{7}(\mathrm{a}), \mathrm{Sr}_{3} \mathrm{Fe}_{2} \mathrm{O}_{6}$ (b) and $\mathrm{Sr}_{3} \mathrm{Fe}_{2} \mathrm{O}_{5}$ (c)

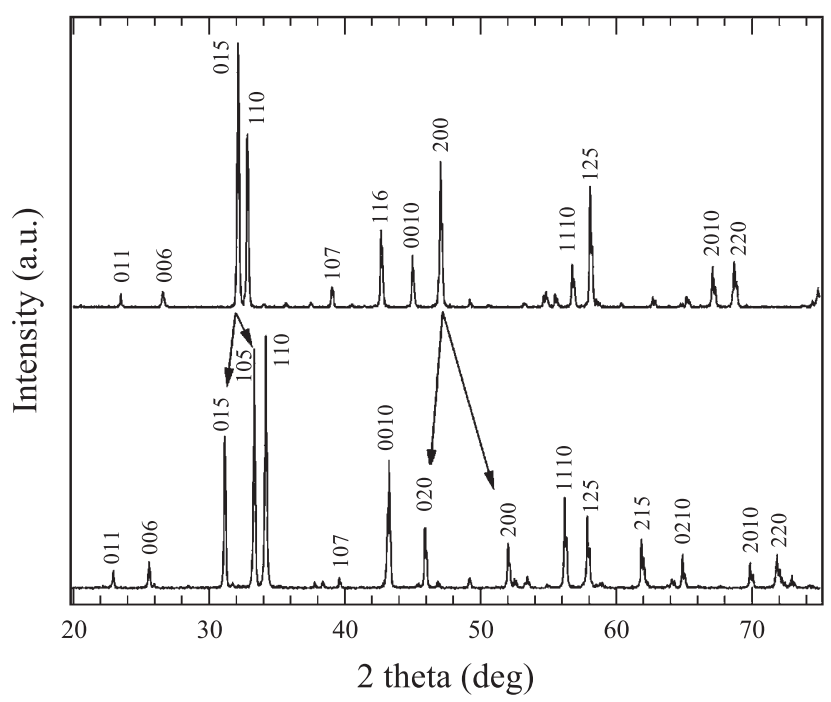

Fig.4 X-ray diffraction patterns of $\mathrm{Sr}_{3} \mathrm{Fe}_{2} \mathrm{O}_{7}$ (up) and $\mathrm{Sr}_{3} \mathrm{Fe}_{2} \mathrm{O}_{5}$ (down).

This new structure can be considered as a two-leg spin ladder system $(n=2)$. Spin ladder system has been widely studied as theories predict that the ground state of an $S=1 / 2$ ladder is a gapped singlet spin liquid state when the $n$ number is even but is a gapless singlet spin liquid state when $n$ is odd. Therefore, it is interesting to compare the copper(II) and iron(II) ladder systems $\mathrm{Sr}_{n-1} \mathrm{Cu}_{n} \mathrm{O}_{2 n-1}$ and $\mathrm{Sr}_{n+1} \mathrm{Fe}_{n} \mathrm{O}_{2 n+1}$. In the former, the ladders share their $\mathrm{O}-\mathrm{O}$ edges to form dense $\mathrm{Cu}_{n} \mathrm{O}_{2 n-1}$ sheets. The iron(II) ladders, on the other hand, are dispersed in the $I$-centered orthorhombic lattice with their legs and rungs oriented along the $b$ and $c$ axes, respectively.

Mössbauer spectroscopy as well as neutron diffraction showed no magnetic order at room temperature and that at $4 \mathrm{~K}$ iron is in the same state as in $\mathrm{SrFeO}_{2}$. Magnetic moments are aligned parallel to the $c$ axis and the magnetic structure is a $G$-type antiferromagnetic structure, the same spin
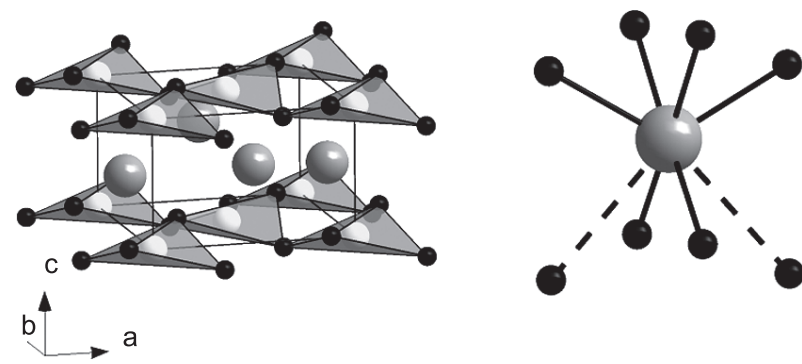

Fig.5 Schematic view of $\mathrm{CaFeO}_{2}$ (a) and the oxygen coordination of calcium (b).

transition as found in $\mathrm{Sr}_{3} \mathrm{Mn}_{2} \mathrm{O}_{7}{ }^{20)}$.

It is highly desirable to extend this family of compounds with respect to $n$ to get a better understanding of the magnetic properties of these materials.

\section{$4 \mathrm{CaFeO}_{2}$}

The stability of $\mathrm{SrFeO}_{2}$ against cation substitution was shown by the synthesis of the solid solution $\mathrm{Sr}_{1-x} \mathrm{Ca}_{x} \mathrm{FeO}_{2}$ $(0 \leq x \leq 1)$. Powder XRD pattern showed that the structure was very similar to $\mathrm{SrFeO}_{2}$. However, the replacement of the interlayer cation resulted in longer reduction time for $\mathrm{Ca}$ rich phases. The lattice parameters exhibited a linear evolution from $\mathrm{SrFeO}_{2}$ to $\mathrm{Sr}_{0.2} \mathrm{Ca}_{0.8} \mathrm{FeO}_{2}$ and a structural phase transition around $x=0.8^{21}$. For the end member $\mathrm{CaFeO}_{2}$, we were able to observe additional diffraction peaks showing the formation of a $\sqrt{2} a_{\mathrm{p}} \times \sqrt{2} a_{\mathrm{p}} \times c_{\mathrm{p}}$ superlattice ${ }^{22)}$. In this structure, the infinite $\mathrm{FeO}_{2}$ layers contain $\mathrm{FeO}_{4}$ units which distort heavily from square-planar toward tetrahedra and rotate along the $c$-axis. It is well known that perovskite structure $A B \mathrm{O}_{3}$ are governed solely by the size of the $A$ site. When the so-called Goldschmidt factor $t$ is close to 1 as in $\mathrm{SrFeO}_{3}$, one obtains an ideal structure with the cubic space group, and the $A$ site is 12 -fold coordinated by oxygen atoms (Fig. 1a). When $t$ deviates from 1, tilting or rotating $B \mathrm{O}_{6}$ octahedra of various types occur, leading to lowered coordination numbers, lowered symmetries, and superstructures. In such cases, the $\mathrm{BO}_{6}$ octahedra are considered to be rigid and preserve their shape upon rotations. In $\mathrm{SrFeO}_{2}$, strontium occupies an 8 -fold site within the iron square planar framework. In $\mathrm{CaFeO}_{2}$, calcium has a 6-fold coordination in reason of the $\mathrm{FeO}_{4}$ units which experience both tilting and distorting (Fig. 5b).

Such lattice distortions usually strongly affect the magnetic interactions via the rupture of the perfect $\angle M-\mathrm{O}$ $M=180^{\circ}$ angle. The simple substitution of strontium by calcium in $\mathrm{SrFeO}_{2}$ should increase the Néel Temperature $\left(T_{\mathrm{N}}\right)$ in reason of shorter $\mathrm{Fe}-\mathrm{Fe}$ distances. In this case, the 
$\angle \mathrm{Fe}-\mathrm{O}$-Fe change from $180^{\circ}$ to $160^{\circ}(-11 \%)$ and the $T_{\mathrm{N}}$ drops from $470 \mathrm{~K}$ to $420 \mathrm{~K}(-11 \%)$. This slight change is remarkable; for instance the Néel temperature of the wellstudied perovskite system $A \mathrm{MnO}_{3}(A=\mathrm{La}$ and rare earth elements) is drastically modified $(140 \mathrm{~K}$ for La and $25 \mathrm{~K}$ for $\mathrm{Gd}$ ) by small angle changes $\left(155.1^{\circ}\right.$ for $\mathrm{La}$ and $146.2^{\circ}$ for $\mathrm{Gd})$. This contrasting behavior of $\mathrm{CaFeO}_{2}$ is explained by the strong hybridization of the $\mathrm{Fe}-d_{x^{2}-y^{2}}$ and the $\mathrm{O}-p_{\sigma}$ orbital are barely influenced by the bridging angle. The $\mathrm{CaFeO}_{2}$ structure shows how $\mathrm{FeO}_{4}$ can be flexible to accommodate its environment and further investigation against temperature and substitutions are ongoing in order to observe the limits of this coordination.

\section{Conclusion}

Unlike the case of cuprates, the coordination geometries in iron oxides were almost exclusively restricted to 3D polyhedra. The use of $\mathrm{CaH}_{2}$ has allowed us to overcome these restrictions and to open new doors toward novel iron oxides with square planar coordination and therefore new chemical and physical properties. These new materials are remarkable in reason of their flexibility and capability of oxygen uptake and release. It would be interesting to test hydride reduction on other iron oxides to create new members of spin ladders (Fig. 6) and also on other transition metal oxides to possibly create novel coordinations and overcome oxygen content limits.

\section{Acknowledgement}

This work was supported by a Grant-in-Aid for Science Research on Priority Areas "Novel States of Matter Induced by Frustration" (No. 19052004) from the Ministry of Education, Culture, Sports, Science and Technology of Japan and by the Global COE program International Center for Integrated Research and Advanced Education in Materials Science, Kyoto University, Japan. One of the authors (Y.T.) was supported by the Japan Society for the Promotion of Science for Young Scientists. The authors wish to thank Takashi Watanabe, Naoaki Hayashi, Werner Paulus, Takateru Kawakami and Mikio Takano for their genuinely helpful advices and discussions.

\section{References}

1) Z. Shao and S.M. Haile: "A high-performance cathode for the next generation of solid-state fuel cells", Nature, 431(2004) 170-173.

2) S.P.S. Badwal and F.T. Ciacchi: "Ceramic membrane technologies for oxygen separation", Adv. Mater., 13 (2001) 993 .
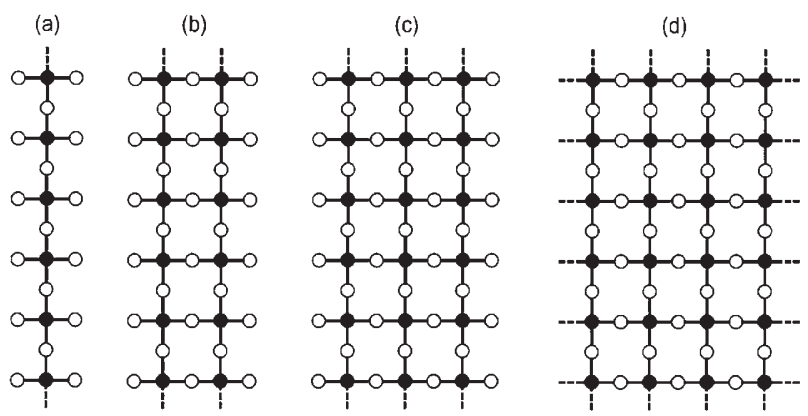

Fig.6 Schematic representation of $n$-legged spin ladders in structures containing square planes. $n=1$ i.e. one dimensional chain (a), $n=2$ corresponding to $\mathrm{Sr}_{3} \mathrm{Fe}_{2} \mathrm{O}_{5}$ (b), $n=3$ corresponding to the hypothetical $\mathrm{Sr}_{4} \mathrm{Fe}_{3} \mathrm{O}_{7}$ (c), $n=\infty$ corresponding to $\mathrm{SrFeO}_{2}$ (d).

3) H. Falcon, J.A. Barbero, J.A. Alonso, J.L. G. Fierro, and M.J. Martinez-Lope: " $\mathrm{SrFeO}_{3-\delta}$ perovskite oxides: chemical features and performance for methane combustion" Chem. Mat., 14(2002)2325-2333.

4) Y.D. Wang, J.B. Chen, and X.H. Wu: "Preparation and gas-sensing properties of perovskite-type $\mathrm{SrFeO}_{3}$ oxide", Mater. Lett., 49(2001)361-364.

5) Y. Takeda, K. Kanno, T. Takada, and O. Yamamoto: "Phase relation in the oxygen nonstoichiometric system $\mathrm{SrFeO}_{x}(2.5 \leq x \leq 3)$ ", Solid-State Chem., 63(1986)237249.

6) J.P. Hodges, S. Short, J.D. Jorgensen, X. Xiong, B. Dabrowski, S.M. Mini, and C.W. Kimball: "Evolution of oxygen-vacancy ordered crystal structures in the perovskite series $\operatorname{Sr}_{n} \mathrm{Fe}_{n} \mathrm{O}_{3 n-1}(n=2,4,8$, and $\infty)$, and the relationship to electronic and magnetic properties", J. Solid-State Chem., 151(2000) 190-209.

7) J.-C. Grenier, A. Wattiaux, J.-P. Doumerc, P. Dordor, L. Fournes, J.-P. Chaminade, and M. Pouchard: "Electrochemical oxygen intercalation into oxide networks", J. Solid State Chem., 96(1996)20-30.

8) N. Hayashi, T. Terashima, and T. Takano: "Oxygenholes creating different electronic phases in $\mathrm{Fe}^{4+}$ oxides: successful growth of single crystalline films of $\mathrm{SrFeO}_{3}$ and related perovskites at low oxygen pressure", J. Mater. Chem., 11(2001)2235-2237.

9) M.A. Hayward and M.J. Rosseinsky: "Anion Vacancy Distribution and Magnetism in the New Reduced Layered $\mathrm{Co}(\mathrm{II}) / \mathrm{Co}$ (I) Phase $\mathrm{LaSrCoO}_{3.5-x}$ ", 12(2000) 2182-2195.

10) M.A. Hayward: "Phase Separation during the Topotactic Reduction of the Pyrochlore $\mathrm{Y}_{2} \mathrm{Ti}_{2} \mathrm{O}_{7}$ ", Chem. Mater., 17(2005)670-675.

11) Y. Tsujimoto, C. Tassel, N. Hayashi, T. Watanabe, H. Kageyama, K. Yoshimura, M. Takano, M. Ceretti, 
C. Ritter, and W. Paulus: "Infinite-layer iron oxide with a square-planar coordination", Nature, 450(2008) 1062-1065.

12) R.M. Hazen and C.W. Burnham: "The crystal structures of gillespite I and II: a structural determination at high pressure", Am. Mineral., 59(1974)1166-1176.

13) K. Leinenweber, J. Linton, A. Navrotsky, Y. Fei, and J.B. Parise: "High-pressure perovskites on the join $\mathrm{CaTiO}_{3}-\mathrm{FeTiO}_{3}$ ", Phys. Chem. Mineral., 22(1995) 251-258.

14) J.M. Pruneda, J. Íñigues, E. Canadell, H. Kageyama, and M. Takano: "Structure and Electronic Properties of $\mathrm{SrFeO}_{2}$ First Principles", Phys. Rev. B, 78(2008) 115101.

15) H.J. Xiang, S.H. Wei, and M.H. Whangbo: "Origin of the structural and magnetic anomalies of the layered compound $\mathrm{SrFeO}_{2}$ : a density functional investigation", Phys. Rev. Lett., 100(2008) 167207.

16) T. Kawakami, Y. Tsujimoto, H. Kageyama, Xing-Qiu Chen, C.L. Fu, C. Tassel, A. Kitada, S. Suto, K. Hirama, Y. Sekiya, Y. Makino, T. Okada, T. Yagi, N. Hayashi, K. Yoshimura, S. Nasu, R. Podloucky, and M. Takano: "Spin transition in a four-coordinate iron oxide", Nature Chemistry, 1(2009)371-376.

17) S. Ju and C.-T. Yi: "Giant optical anisotropy in an infinite-layer iron oxide $\mathrm{SrFeO}_{2}$ : An ab initio investigation", Applied Physics Letters, 94(2009)
061902.

18) S. Inoue, M. Kawai, Y. Shimakawa, M. Mizumaki, N. Kawamura, T. Watanabe, Y. Tsujimoto, H. Kageyama, and $\mathrm{K}$. Yoshimura: "Single-crystal epitaxial thin films of $\mathrm{SrFeO}_{2}$ with $\mathrm{FeO}_{2}$ "infinite layers"', Applied Physics Letters, 92(2008) 161911.

19) H. Kageyama, T. Watanabe, Y. Tsujimoto, A. Kitada, Y. Sumida, K. Kanamori, K. Yoshimura, N. Hayashi, S. Muranaka, M. Takano, M. Ceretti, W. Paulus, C. Ritter, and G. André: "Spin-Ladder Iron Oxide: $\mathrm{Sr}_{3} \mathrm{Fe}_{2} \mathrm{O}_{5}$ ", Angewandte Chemie, 47(2008)5740-5745.

20) J.F. Mitchell, J.E. Millburn, M. Medarde, S. Short, J.D. Jorgensen, and M.T. Fernández-Díaz: " $\mathrm{Sr}_{3} \mathrm{Mn}_{2} \mathrm{O}_{7}$ : $\mathrm{Mn}^{4+}$ parent compound of the $n=2$ layered CMR manganites", J. Solid State Chem., 141(1998)599-603.

21) C. Tassel, T. Watanabe, Y. Tsujimoto, N. Hayashi, A. Kitada, Y. Sumida, T. Yamamoto, H. Kageyama, M. Takano, and K. Yoshimura: "Stability of the Infinite Layer Structure with Iron Square Planar Coordination", J. Am. Chem. Soc., 130 (2008)3764-3765.

22) C. Tassel, J.-M. Pruneda, N. Hayashi, T. Watanabe, A. Kitada, Y. Tsujimoto, H. Kageyama, K. Yoshimura, M. Takano, M. Nishi, K. Ohoyama, M. Mizumaki, N. Kawamura, J. Íñiguez, and E. Canadell: " $\mathrm{CaFeO}_{2}$ : A New Type of Layered Structure with Iron in a Distorted Square Planar Coordination", J. Am. Chem. Soc., 131 (2009) 221-229. 